\title{
Respostas de culturas à adubação sulfatada e deposição de enxofre atmosférico ${ }^{1}$
}

\author{
Tales Tiecher ${ }^{2}$, Danilo Rheinheimer dos Santos ${ }^{3}$, Jimmy Walter Rasche Alvarez², Fábio Joel Kochem Mallmann ${ }^{3}$, \\ Rogério Piccin ${ }^{4}$, Gustavo Brunetto ${ }^{3}$
}

\begin{abstract}
RESUMO
Solos com baixo teor de argila e matéria orgânica apresentam baixa disponibilidade de enxofre (S) e, por isso, as culturas podem responder à adubação sulfatada. No entanto, a mobilidade de $\mathrm{S}$ no perfil do solo e sua deposição atmosférica pela água da chuva dificultam o estabelecimento do nível de suficiência do nutriente no solo. Este trabalho objetivou avaliar a resposta de culturas à adubação sulfatada e quantificar o $\mathrm{S}$ atmosférico depositado no solo pela água da chuva. Os cultivos avaliados (mamoneira, trigo, feijão-de-porco e milheto) foram realizados entre 2006 e 2009, em um Argissolo Vermelho distrófico arênico. As doses de S aplicadas em cada cultivo foram de $0,5,10$ e $20 \mathrm{~kg} \mathrm{ha}^{-1}$, utilizando-se gesso agrícola como fonte de S. Avaliaram-se a produção de matéria seca e o acúmulo de S na parte aérea do milheto e do feijão-de-porco, e a produção e o teor de $S$ nos grãos de mamona e de trigo. Determinou-se o teor de $\mathrm{S}$ disponível no solo até $60 \mathrm{~cm}$ de profundidade e a deposição de $\mathrm{S}$ pelas precipitações. A maior produção de grãos da mamona e de matéria seca do segundo cultivo de feijão-de-porco foi obtida com aplicação de 14,5 $\pm 0,35 \mathrm{~kg} \mathrm{ha}^{-1}$ de $\mathrm{S}$. Mesmo com teores de $\mathrm{S}$ abaixo dos níveis de suficiência, não houve respostas do trigo, do milheto e do primeiro cultivo de feijão-de-porco à adubação sulfatada. $\mathrm{O}$ aporte de $\mathrm{S}$ atmosférico ao solo foi de $4,5 \mathrm{~kg} \mathrm{ha}^{-1}$ ano $^{-1}$ e pode ter contribuído para a ausência de resposta desses cultivos.
\end{abstract}

Palavras-chave: disponibilidade de enxofre, nível de suficiência de enxofre, biociclagem de enxofre, gesso agrícola.

\section{ABSTRACT}

\section{Crops response to sulfur fertilization and atmospheric deposition}

Soils with low levels of clay and organic matter have low sulfur (S) availability. Therefore, crop yields may increase due to $S$ fertilization. However, the mobility of $S$ in the soil profile and its atmospheric deposition by rainwater make it difficult to establish the S sufficiency level in the soil. The objective of this study was to evaluate the response of crops to $S$ fertilization and quantify the $S$ atmospheric deposition in the soil via rainwater. The cultivations evaluated (castor bean, wheat, jack bean and millet) were performed between 2006 and 2009 in a Hapludalf. The S doses applied in each cultivation were $0,5,10$ and $20 \mathrm{~kg} \mathrm{ha}^{-1}$, using gypsum as a S source. The dry matter production and $\mathrm{S}$ accumulation were evaluated the in the shoots of millet and jack bean, and production and the $\mathrm{S}$ content were evaluated in castor bean and wheat. We also determined the level of available $S$ in the soil to a depth of $60 \mathrm{~cm}$ and the $S$ deposition by rainfall. The

\footnotetext{
Recebido para publicação em 18/07/2012 e aprovado em 08/03/2013.

${ }^{1}$ Pesquisa financiada pelo CNPq.

'Engenheiro-Agrônomo, Mestre. Departamento de Solos, Universidade Federal de Santa Maria, Avenida Roraima, 1000, Bairro Camobi, 97105-900, Santa Maria, Rio Grande do Sul, Brasil. tales.t@hotmail.com (autor para correspondência); jwrasche@yahoo.com.br;

${ }^{3}$ Engenheiro-Agrônomo, Doutor. Departamento de Solos, Universidade Federal de Santa Maria, Avenida Roraima, 1000, Bairro Camobi, 97105-900, Santa Maria, Rio Grande do Sul, Brasil.danilonesaf@gmail.com (Bolsista do CNPq); fabiojkmallmann@gmail.com (Bolsista CAPES); brunetto.gustavo@gmail.com

${ }^{4}$ Graduando em Agronomia. Departamento de Solos, Universidade Federal de Santa Maria, Avenida Roraima, 1000, Bairro Camobi, 97105-900, Santa Maria, Rio Grande do SuI, Brasil.rogeriopiccin@hotmail.com (Bolsista do CNPq).
}

Rev. Ceres, Viçosa, v. 60, n.3, p. 420-427, mai/jun, 2013 
highest grain yield of castor bean and the highest dry matter yield in the second cultivation of jack bean were obtained with the application of $14.5 \pm 0.35 \mathrm{~kg} \mathrm{ha}^{-1} \mathrm{~S}$. Even with $\mathrm{S}$ content below the sufficiency levels, there was no response of wheat, millet and the first crop of jack bean to the $\mathrm{S}$ fertilization. The atmospheric input of $\mathrm{S}$ to the soil was $4.5 \mathrm{~kg}^{-}$ ${ }^{1}$ year $^{-1}$ and it may have contributed to the no response of these crops.

Key words: sulfur availability, sulfur sufficiency level, sulfur biocycling, gypsum.

\section{INTRODUÇÃO}

No solo, até $98 \%$ do enxofre pode ser encontrado na forma orgânica (Brady \& Weil, 2002). Por isso, a capacidade do solo de suprir a demanda da planta pelo nutriente está intimamente relacionada com o teor de matéria orgânica do solo e à mineralização do $\mathrm{S}$ orgânico para formas inorgânicas, como o sulfato. Na solução do solo, o sulfato é facilmente lixiviado, pois é fracamente retido por meio do mecanismo de adsorção por coordenação (Essington, 2005; Ishiguro \& Makino, 2011), sendo que a sua adsorção é maior em solos mais argilosos e ricos em óxidos de ferro e alumínio (Pozza et al., 2009; Jung et al., 2011). Por essa razão, solos com baixos teores de matéria orgânica, de argila e de óxidos, como aqueles com textura arenosa, apresentam, teoricamente, maior probabilidade de resposta das culturas à aplicação de $\mathrm{S}$.

A deficiência de $\mathrm{S}$ pode ainda ser potencializada quando plantas exigentes por este nutriente, como as fabáceas, brassicáceas e liliáceas (CQFS-RS/SC, 2004) são cultivadas em sistemas de cultivo com alta produtividade e que utilizam fertilizantes minerais concentrados com baixos teores de S. Entretanto, não existe uma condição específica que determine a resposta das culturas à aplicação de $\mathrm{S}$. Exemplo disso é a cultura do trigo (Triticum aestivum), que, apesar de ser uma espécie teoricamente menos exigente em S, pode aumentar sua produção de grãos com a fertilização sulfatada (Barraco et al., 2009; Salvagiotti et al., 2009; Duggan et al., 2010). Contudo, em alguns trabalhos recentes desenvolvidos no sul do Brasil, foi observada ausência de resposta do trigo à aplicação de $\mathrm{S}$ (Rheinheimer et al., 2005; Osório Filho et al., 2007), que pode ser atribuída à rápida lixiviação do sulfato aplicado na semeadura para além da zona de absorção das raízes, e/ou à deposição atmosférica de $\mathrm{S}$, que, no Brasil, varia de $1,12 \mathrm{~kg} \mathrm{ha}^{-1}$, em áreas distantes de zonas urbanas (Araujo, 2011), até 99,2 $\mathrm{kg} \mathrm{ha}^{-1}$, em regiões metropolitanas (Guedes, 1985).

A mamoneira (Ricinus communis), da família euforbiácea, é uma cultura emergente no cenário agrícola nacional, que passou a ser amplamente cultivada nos últimos anos para a produção de biodiesel. Por isso, vários trabalhos já foram desenvolvidos para avaliar a resposta da mamoneira à adubação com macro e micronutrientes (Severino et al., 2005; Severino et al., 2006; Lavres Junior et al., 2012; Silva et al., 2012), mas poucos deles têm dado importância à adubação sulfatada para essa cultura. De acordo com Lavres Júnior et al. (2005), em experimento realizado em casa de vegetação, a mamoneira pode ser uma cultura exigente em $\mathrm{S}$, pois o nutriente foi o terceiro elemento que mais restringiu a produção de matéria seca da cultura. Somado a isso, informações sobre a exigência de $\mathrm{S}$ de outras espécies amplamente difundidas na agricultura brasileira, como as plantas de cobertura de solo, dentre elas o feijão-de-porco (Canavalia ensiformis) e o milheto (Pennisetum americanum), também são escassas.

Os objetivos deste trabalho foram: (i) avaliar a resposta da mamoneira, do trigo, do feijão-de-porco e do milheto à adubação sulfatada e sua relação com o teor de $\mathrm{S}$ disponível no solo; (ii) contabilizar a quantidade de S atmosférico depositado no solo pela água da chuva.

\section{MATERIAL E MÉTODOS}

O experimento foi realizado entre novembro de $2006 \mathrm{e}$ março de 2009, em Santa Maria (RS), em um Argissolo Vermelho distrófico arênico (EMBRAPA, 2006), intensivamente cultivado sob sistema de preparo convencional nas décadas de 60 e 70. Entre meados da década de 70 até 1994, o solo não foi cultivado, havendo crescimento de plantas espontâneas. Em 1994, aplicaram-se 6,4 t ha-1 de calcário dolomítico (PRNT 100\%) para elevar o pH do solo, em água, até 6,0, e $140 \mathrm{~kg} \mathrm{ha}^{-1}$ de $\mathrm{P}_{2} \mathrm{O}_{5}$, a lanço, na forma de superfosfato triplo, para elevar a disponibilidade de fósforo acima do nível de suficiência (CQFS-RS/SC, 2004), com posterior incorporação com uma aração e duas gradagens. Após estas operações, foi adotado o sistema plantio direto na área. O clima da região é Cfa (subtropical úmido), segundo classificação de Köppen, com precipitação e temperatura média anual de $1.769 \mathrm{~mm}$ e $19,2{ }^{\circ} \mathrm{C}$, respectivamente.

Antes da instalação do experimento, o solo da camada de 0-10 cm apresentava: $140 \mathrm{~g} \mathrm{~kg}^{-1}$ de argila; $12,8 \mathrm{~g} \mathrm{~kg}^{-1} \mathrm{de}$ 
matéria orgânica; pH em água de 5,2; Índice SMP 6,0;3,4, 0,8 e $0,2 \mathrm{cmol}_{\mathrm{c}} \mathrm{kg}^{-1}$ de cálcio, magnésio e alumínio trocáveis, respectivamente; $4,5 \mathrm{mg} \mathrm{kg}^{-1}$ de sulfato disponível (extrator $\left.\mathrm{Ca}\left(\mathrm{H}_{2} \mathrm{PO}_{4}\right)_{2} \cdot \mathrm{H}_{2} \mathrm{O}, 0,008 \mathrm{~mol} \mathrm{~L}^{-1} \mathrm{de} \mathrm{P}\right) ; 20,0 \mathrm{e}$ $44,0 \mathrm{~m} \mathrm{dm}^{-3}$ de fósforo disponível e potássio trocável (extrator Mehlich 1), respectivamente. Antes da instalação do experimento, foi reaplicada $1,6 \mathrm{tha}^{-1}$ de calcário dolomítico (PRNT 100\%) para elevar o pH em água até 5,5, seguindo-se uma escarificação a $30 \mathrm{~cm}$ de profundidade. O delineamento experimental utilizado foi o de blocos ao acaso, com quatro repetições, cada unidade experimental medindo $9,5 \times 11,0 \mathrm{~m}$.

As doses de $\mathrm{S}$ aplicadas em cada cultivo foram de 0,5 , 10 e $20 \mathrm{~kg} \mathrm{ha}^{-1}$, a lanço e utilizando-se gesso agrícola $\left(\mathrm{CaSO}_{4} \cdot 2 \mathrm{H}_{2} \mathrm{O}\right)$. A sequência de espécies cultivadas na área experimental foi: mamoneira (Ricinus communis L.), cultivar AL-Guarany, em consórcio com feijão-de-porco (Canavalia ensiformis), em 2006/07 e 2007/2008, trigo (Triticum aestivum L.) cultivar BRS Tarumã, em 2007, e milheto (Pennisetum americanum), na safra 2008/09. Nos cultivos de mamoneira, as doses de $\mathrm{S}$ foram aplicadas concomitantemente com a semeadura; no cultivo de trigo as doses de $\mathrm{S}$ foram aplicadas no início da floração (80 DAE); no cultivo do milheto, a aplicação das doses de $\mathrm{S}$ foi realizada $20 \mathrm{DAE}$ da cultura.

Nos cultivos de mamoneira, foram aplicados $15 \mathrm{~kg}$ $\mathrm{ha}^{-1} \mathrm{de} \mathrm{N}$ na semeadura e $45 \mathrm{~kg} \mathrm{ha}^{-1} \mathrm{em}$ cobertura, 45 dias após a emergência (DAE), conforme recomendação de Severino et al. (2005). Na semeadura dos cultivos da mamoneira, foram aplicados também $40 \mathrm{~kg} \mathrm{ha}^{-1} \mathrm{de}_{2} \mathrm{O}$, seguindo a recomendação de Moterle et al. (2007). No cultivo do trigo, foram aplicados $15 \mathrm{~kg} \mathrm{ha}^{-1}$ de $\mathrm{N}$ e $20 \mathrm{~kg}$ ha $^{-1}$ de $\mathrm{K}_{2} \mathrm{O}$ na semeadura e $45 \mathrm{~kg} \mathrm{ha}^{-1}$ de $\mathrm{N}$ em cobertura divididos entre os estádios de alongamento e afilhamento, conforme recomendação da CQFS-RS/SC (2004). $\mathrm{Na}$ cultura do milheto, foram aplicados $40 \mathrm{~kg} \mathrm{ha}^{-1}$ de $\mathrm{N}$ no alongamento e $40 \mathrm{~kg} \mathrm{ha}^{-1}$ de $\mathrm{N}$ no afilhamento. A adubação fosfatada não foi realizada em nenhum dos cultivos, pois o teor de P no solo era Muito Alto (97,5 $\mathrm{mg} \mathrm{kg}^{-1}$ ), segundo a CQFS-RS/SC (2004). Em todos os cultivos foi utilizada ureia como fonte de $\mathrm{N}$ e cloreto de potássio como fonte de $\mathrm{K}$.

Os cultivos de mamoneira foram realizados num espaçamento de 1,40 x 0,80m. Aos 52 DAE da mamoneira, no primeiro cultivo, e aos sete DAE, no segundo cultivo, foi semeado o feijão-de-porco, na entrelinha da mamoneira, em linhas duplas distantes $0,40 \mathrm{~m}$ entre si, com espaçamento de $0,40 \mathrm{~m}$ na linha, e distantes $0,50 \mathrm{~m}$ das linhas de mamoneira. A parte aérea do feijão-de-porco foi coletada aos 75 e 50 DAE da cultura, no primeiro e segundo cultivos, respectivamente, numa área útil de $11,76 \mathrm{~m}^{2}$ (duas fileiras de $8,4 \mathrm{~m}$ em cada parcela). Após a coleta da parte aérea, o restante do feijão-de-porco foi roçado e os resí- duos permaneceram na superfície do solo. Em ambos os cultivos, a colheita da mamoneira iniciou-se aos $150 \mathrm{DAE}$, com colheitas semanais até o fim do ciclo da cultura, numa área útil de $50 \mathrm{~m}^{2}$. A colheita do trigo foi realizada em uma área útil de $12 \mathrm{~m}^{2}$. A parte aérea do milheto foi coletada aos 53 DAE, em uma área útil de $6 \mathrm{~m}^{2}$.

As partes aéreas dos cultivos de feijão-de-porco e de milheto foram secadas em estufa com circulação forçada de ar a $\pm 65^{\circ} \mathrm{C}$ até massas constantes e, em seguida, a matéria seca de cada espécie foi quantificada. As produções de grãos de mamona e de trigo foram corrigidas para 13\% de umidade e, após secagem, os grãos foram moídos. Amostras moídas do material seco de tecido das partes aéreas e de grãos foram submetidas à digestão completa com ácido nítrico e ácido perclórico (Tabatabai \& Bremnem, 1970), com posterior determinação do S total por turbidimetria (Tedesco et al., 1995). Nos cultivos de feijão-de-porco, também foi determinado o teor de $\mathrm{N}$ total na matéria seca da parte aérea, seguindo procedimento proposto por Tedesco et al. (1995). Além disso, no cultivo de trigo foi determinada a massa hectolítrica de grãos.

Amostras estratificadas de solo foram coletadas nas camadas de 0-10, 10-20, 20-30, 30-40, 40-50 e 50-60 cm na floração do trigo (03/10/2007, correspondendo a 40 dias após a aplicação de S) e, após o segundo cultivo de mamoneira (16/07/2008, correspondendo a 240 dias após a aplicação de S), em uma trincheira de $0,3 \times 0,3$ x 0,6 m aberta em cada parcela. $\mathrm{O}$ solo das amostras foi secado em estufa com circulação forçada de ar a $\pm 65^{\circ} \mathrm{C}$, moído, passado em peneira com malha de $2 \mathrm{~mm}$ e, em seguida, foi submetido à extração de $\mathrm{S}$ disponível pelo extrator de $\mathrm{Ca}\left(\mathrm{H}_{2} \mathrm{PO}_{4}\right)_{2}\left(0,008 \mathrm{~mol} \mathrm{~L}^{-1} \mathrm{de} \mathrm{P}\right)$ (Tabatabai \& Bremnem, 1970). A concentração de $S$ foi determinada por turbidimetria (Tedesco et al., 1995).

As amostras de água das precipitações e das irrigações foram coletadas entre setembro de 2007 e abril de 2009, utilizando-se pluviômetros do tipo Ville de Paris instalados no interior do experimento. As amostras de água das chuvas e da irrigação foram filtradas em membrana de celulose $0,22 \mu \mathrm{m}$ e submetidas à determinação do teor de $\mathrm{SO}_{4}^{-2}$ por análise de cromatografia líquida de alta eficiência (HPLC). Os valores de precipitação foram obtidos na Estação Climatológica da Universidade Federal de Santa Maria.

Os dados de produção de matéria seca e de grãos, do teor total de $\mathrm{S}$ nos tecidos e nos grãos e da quantidade de $S$ acumulado na parte aérea das plantas e exportada pelos grãos, foram submetidos à análise de variância, utilizando-se o modelo estatístico de blocos ao acaso. Quando significativas, as médias foram ajustadas por regressões polinomiais de até segundo grau, em função das doses de S aplicadas. Já os dados de sulfato extraído do solo foram 
analisados segundo um esquema bifatorial (doses de sulfato e camadas de solo), considerando-se a não aleatoriedade das camadas de solo, utilizando-se o seguinte modelo estatístico:

$$
\begin{aligned}
& Y_{i j k}=\mu+B_{i}+D_{j}+\operatorname{erro} a(i, j)+C_{k}+\operatorname{errob}(i, k)+ \\
& D C_{j k}+\operatorname{erroc}(i, j, k),
\end{aligned}
$$

em que $\mu=$ média geral do experimento; $B=\operatorname{blocos}(i=1$, $2,3,4) ; D=$ dose de $\mathrm{S}$ aplicada $(j=1,2,3,4) ; C=$ camada de solo $(k=1,2,3,4,5,6)$ e erro $=$ erro experimental.

\section{RESULTADOS E DISCUSSÃO}

A aplicação das doses de $\mathrm{S}$ na forma de gesso agrícola não alterou o teor de $\mathrm{S}$, em nenhuma das camadas de solo amostradas nas duas avaliações (Tabela 1). Nas duas coletas de solo, os teores de $\mathrm{S}$ na camada $0-10 \mathrm{~cm}$ foram menores que o nível de suficiência para as culturas menos exigentes, como as gramíneas $\left(5,0 \mathrm{mg} \mathrm{dm}^{-3}\right)$, e para as culturas mais exigentes, como as leguminosas (10 $\mathrm{mg} \mathrm{dm}^{-3}$ ) (CQFS-RS/SC, 2004), sendo que a média de todos os tratamentos foi de $4,0 \mathrm{mg} \mathrm{dm}^{-3}$, na primeira coleta, e de 4,7 $\mathrm{mg} \mathrm{dm}^{-3}$, na segunda. Isso demonstra que, mesmo amostrando o solo 40 dias após a aplicação de $\mathrm{S}$, com uma precipitação total de 243,4 mm durante esse período (24/08/2007 a 03/10/2007), o extrator $\mathrm{Ca}\left(\mathrm{H}_{2} \mathrm{PO}_{4}\right)_{2}$ não foi sensível para detectar as flutuações nos teores de $\mathrm{S}$ do solo, decorrentes da aplicação de fertilizante sulfatado, corroborando com os resultados obtidos por Osório Filho et al. (2009).

O teor total e a exportação de S nos grãos da mamona apresentaram aumento quadrático com a aplicação das

Tabela 1. Teor de $\mathrm{S}$ disponível em seis camadas de um Argissolo Vermelho distrófico arênico, na floração do trigo e após o segundo cultivo da mamoneira, após aplicação de doses de 5 a $20 \mathrm{~kg} \mathrm{ha}^{-1}$

\begin{tabular}{|c|c|c|}
\hline \multirow{2}{*}{$\begin{array}{l}\text { Camada de solo } \\
\mathrm{cm}\end{array}$} & $\begin{array}{c}\text { Floração do } \\
\text { trigo }^{1}\end{array}$ & $\begin{array}{l}\text { Após } 2^{\circ} \text { cultivo } \\
\text { da mamoneira }\end{array}$ \\
\hline & \multicolumn{2}{|c|}{ Teor de $\mathrm{S}$ disponível $\left(\mathrm{mg} \mathrm{dm}^{-3}\right)$} \\
\hline $0-10$ & $4,0 \mathrm{a}$ & $4,7 \mathrm{a}$ \\
\hline $10-20$ & $2,7 \mathrm{~b}$ & $3,4 \mathrm{~b}$ \\
\hline $20-30$ & $2,3 \mathrm{c}$ & $2,6 \mathrm{c}$ \\
\hline $30-40$ & $1,8 \mathrm{~d}$ & $2,2 \mathrm{~d}$ \\
\hline $40-50$ & $1,7 \mathrm{~d}$ & $1,9 \mathrm{~d}$ \\
\hline $50-60$ & $1,4 \mathrm{~d}$ & $1,7 \mathrm{~d}$ \\
\hline Fontes de variação & $\operatorname{Pr}>F$ & $\operatorname{Pr}>F$ \\
\hline Dose & ns & ns \\
\hline Camada & $* * *$ & $* * *$ \\
\hline Dose*Camada & $\mathrm{ns}$ & $\mathrm{ns}$ \\
\hline $\mathrm{CV}(\%)$ & 13,85 & 12,41 \\
\hline
\end{tabular}
de $\mathrm{S}$

${ }^{1}$ Coleta realizada 40 dias após a aplicação de $\mathrm{S} ;{ }^{2}$ Coleta realizada 240 dias após a aplicação de S; Médias seguidas pela mesma letra na coluna não diferem entre si a $5 \%$ de probabilidade pelo teste de Tukey; ns = não significativo; $* * *$ significativo a $P<0,001$. doses de S nos dois anos de avaliação (Tabela 2). O maior teor de $\mathrm{S}$ nos grãos de mamona foi obtido com a aplicação de 18,2 e 17,0 $\mathrm{kg} \mathrm{ha}^{-1}$ de $\mathrm{S}$ no primeiro e segundo cultivos, respectivamente. Já a maior quantidade de $\mathrm{S}$ exportado nos grãos de mamona foi obtida com a aplicação de 15,5 e $17,0 \mathrm{~kg} \mathrm{ha}^{-1}$ de $\mathrm{S}$ no primeiro e segundo cultivos, respectivamente. A produção da mamona não foi afetada a 5\% de significância, mas a 10\% de significância foi verificado aumento quadrático da produção de grãos com a adubação sulfatada nos dois cultivos. Esse nível de significância mais elevado para avaliar a produção da cultura pode ser justificado pelo fato de que os cultivares de mamoneira, como a AL-Guarany, possuem ainda grande variabilidade genética, o que pode mascarar o efeito dos tratamentos. Considerando-se a significância de $10 \%$, a maior produção de grãos de mamona foi obtida com a aplicação de 14,1 e $14,8 \mathrm{~kg} \mathrm{ha}^{-1}$ de $\mathrm{S}$ no primeiro e no segundo cultivos, respectivamente. Com base nesses resultados, evidencia-se que esta cultura é uma espécie exigente em $\mathrm{S}$, assim como demonstrado por Lavres Junior et al. (2005), que, em estudo de casa de vegetação, verificaram a redução de até $41 \%$ na produção de matéria seca, quando submetida à condição de deficiência de $\mathrm{S}$.

A produção de matéria seca da parte aérea do feijão-de-porco, em consórcio com a mamoneira, apresentou aumento quadrático com aumento da dose de $\mathrm{S}$ aplicada apenas no segundo cultivo (Tabela 2), mesmo com os teores de $\mathrm{S}$ disponível abaixo do nível crítico para as leguminosas (10 $\mathrm{mg} \mathrm{dm}^{-3}$, segundo a CQFS-RS/ $\mathrm{SC}, 2004)$. Nesse cultivo, a maior produção de matéria seca da parte aérea foi obtida com a aplicação de 14,6 $\mathrm{kg} \mathrm{ha}^{-1}$ de $\mathrm{S}$. Isto pode ter ocorrido por causa de sua implantação logo após a aplicação das doses de S (7 dias), diferentemente do primeiro cultivo dessa cultura, que foi semeada 53 dias após a aplicação de S. O teor total de $\mathrm{S}$ nos tecidos da parte aérea aumentou com a dose, em ambos os cultivos, mas houve incremento no teor de $\mathrm{S}$ acumulado apenas no segundo cultivo. No segundo cultivo, o aumento quadrático do teor de $S$ na parte aérea em função da dose de $S$ aplicada resultou na diminuição da relação $\mathrm{N}: \mathrm{S}$ no tecido vegetal. Sob deficiência de $\mathrm{S}$, ocorre acúmulo de $\mathrm{N}$ não proteico no tecido das plantas, principalmente nas formas de $\mathrm{N}^{-} \mathrm{NO}_{3}{ }^{-}$e $\mathrm{N}$ orgânico solúvel, causando aumento da relação $\mathrm{N}: \mathrm{S}$, o que pode causar diminuição do crescimento vegetal (Fageria, 2001; Crusciol et al., 2006). Isso pode ser uma das possíveis explicações para a menor produção de matéria seca do feijão-de-porco no tratamento testemunha, no segundo cultivo. O teor e acúmulo de $\mathrm{N}$ na parte aérea do feijão-de-porco não foram afetados pela aplicação de $\mathrm{S}$. A quantidade de $\mathrm{N}$ acumulada foi, em média, de 129 e $59 \mathrm{~kg} \mathrm{ha}^{-1}$ no primeiro e segundo cultivos, respectivamente. 


\begin{tabular}{|c|c|c|c|c|c|c|c|c|c|}
\hline \multirow{2}{*}{ Cultivo/Ano } & \multirow{2}{*}{ Avaliação } & \multicolumn{4}{|c|}{ Dose de $S\left(\mathrm{~kg} \mathrm{ha}^{-1}\right)$} & \multirow{2}{*}{$\operatorname{Pr}>\boldsymbol{F}$} & \multirow{2}{*}{ CV, \% } & \multirow{2}{*}{ Equação de regressão } & \multirow{2}{*}{$\mathbf{R}^{2}$} \\
\hline & & $\mathbf{0}$ & 5 & 10 & 20 & & & & \\
\hline Mamoneira & Grãos (t ha-1) & 1,27 & 1,68 & 1,60 & 1,67 & $*$ & 14,68 & $\mathrm{y}=1,3191+0,0582 \mathrm{x}-0,0021 \mathrm{x}^{2}$ & 0,78 \\
\hline \multirow[t]{2}{*}{$2006 / 07$} & Teor de S $\left(\mathrm{g} \mathrm{kg}^{-1}\right)$ & 1,09 & 1,19 & 1,20 & 1,25 & $* *$ & 4,56 & $y=1,0948+0,0168 x-0,0005 x^{2}$ & 0,93 \\
\hline & Exportação $\mathrm{S}\left(\mathrm{kg} \mathrm{ha}^{-1}\right)$ & 1,38 & 2,01 & 1,92 & 2,11 & $* *$ & 17,59 & $y=1,4472+0,0904 x-0,0029 x^{2}$ & 0,82 \\
\hline \multirow[t]{2}{*}{ Feijão-de porco } & Matéria seca $\left(\mathrm{t} \mathrm{ha}^{-1}\right)$ & 3,45 & 3,56 & 3,54 & 3,17 & ns & 12,22 & - & \\
\hline & Teor de $\mathrm{S}\left(\mathrm{g} \mathrm{kg}^{-1}\right)$ & 1,04 & 1,13 & 1,20 & 1,40 & $* *$ & 12,28 & $\mathrm{y}=1,0351+0,0180 \mathrm{x}$ & 0,99 \\
\hline \multirow[t]{4}{*}{$2006 / 07$} & Teor de $\mathrm{N}\left(\mathrm{g} \mathrm{kg}^{-1}\right)$ & 38,0 & 33,2 & 38,5 & 38,9 & $\mathrm{~ns}$ & 7,70 & - & \\
\hline & S acumulado $\left(\mathrm{kg} \mathrm{ha}^{-1}\right)$ & 3,56 & 4,02 & 4,29 & 4,44 & ns & 18,53 & - & \\
\hline & $\mathrm{N}$ acumulado $\left(\mathrm{kg} \mathrm{ha}^{-1}\right)$ & 133 & 120 & 136 & 126 & $\mathrm{~ns}$ & 15,22 & - & \\
\hline & Relação N/S & 38 & 30 & 32 & 28 & $\mathrm{~ns}$ & 15,55 & - & \\
\hline Trigo & Grãos (t ha' $\left.{ }^{-1}\right)$ & 2,39 & 2,38 & 2,24 & 2,45 & ns & 5,41 & - & \\
\hline \multirow[t]{3}{*}{2007} & Teor de S $\left(\mathrm{g} \mathrm{kg}^{-1}\right)$ & 0,94 & 1,13 & 0,83 & 1,08 & $\mathrm{~ns}$ & 18,75 & - & \\
\hline & Exportação $\mathrm{S}\left(\mathrm{kg} \mathrm{ha}^{-1}\right)$ & 2,25 & 2,70 & 1,86 & 2,65 & $\mathrm{~ns}$ & 20,02 & - & \\
\hline & $\mathrm{MH}^{1}\left(\mathrm{~kg} 100 \mathrm{~L}^{-1}\right)$ & 79 & 79 & 80 & 80 & $\mathrm{~ns}$ & 0,80 & - & \\
\hline Mamoneira & Grãos (t ha-1) & 0,81 & 0,91 & 0,89 & 0,93 & $*$ & 7,63 & $y=0,8208+0,0157 x-0,0005 x^{2}$ & 0,71 \\
\hline \multirow[t]{2}{*}{$2007 / 08$} & Teor de S (\%) & 1,30 & 1,32 & 1,61 & 1,56 & $* *$ & 9,00 & $y=1,2603+0,0384 x-0,0011 x^{2}$ & 0,77 \\
\hline & Exportação $S\left(\mathrm{~kg} \mathrm{ha}^{-1}\right)$ & 1,06 & 1,21 & 1,44 & 1,46 & $* *$ & 13,70 & $y=1,0372+0,0523 x-0,0015 x^{2}$ & 0,96 \\
\hline \multirow[t]{2}{*}{ Feijão-de porco } & Matéria seca $\left(\mathrm{t} \mathrm{ha}^{-1}\right)$ & 1,58 & 1,83 & 1,84 & 1,88 & $* *$ & 7,32 & $y=1,5703+0,0412 x-0,0014 x^{2}$ & 0,91 \\
\hline & Teor de $\mathrm{S}\left(\mathrm{g} \mathrm{kg}^{-1}\right)$ & 1,02 & 1,42 & 1,51 & 1,61 & $* *$ & 18,11 & $y=1,0412+0,0741 x-0,0023 x^{2}$ & 0,97 \\
\hline \multirow[t]{4}{*}{$2007 / 08$} & Teor de $\mathrm{N}\left(\mathrm{g} \mathrm{kg}^{-1}\right)$ & 39,2 & 37,0 & 39,1 & 36,2 & ns & 5,90 & - & \\
\hline & $\mathrm{S}$ acumulado $\left(\mathrm{kg} \mathrm{ha}^{-1}\right)$ & 1,63 & 2,59 & 2,83 & 2,97 & $* *$ & 19,22 & $y=1,6457+0,1815 x-0,0060 x^{2}$ & 0,97 \\
\hline & $\mathrm{N}$ acumulado $\left(\mathrm{kg} \mathrm{ha}^{-1}\right)$ & 55 & 58 & 62 & 59 & $\mathrm{~ns}$ & 8,02 & - & \\
\hline & Relação N/S & 39 & 27 & 27 & 23 & $* *$ & 20,43 & $y=37,733-1,8834 x+0,0585 x^{2}$ & 0,87 \\
\hline Milheto & Matéria seca $\left(\mathrm{t} \mathrm{ha}^{-1}\right)$ & 7,41 & 7,50 & 7,01 & 7,00 & ns & 13,51 & - & \\
\hline \multirow[t]{2}{*}{2009} & Teor de $\mathrm{S}\left(\mathrm{g} \mathrm{kg}^{-1}\right)$ & 0,70 & 1,19 & 1,72 & 2,62 & $* * * *$ & 13,36 & $y=0,7165+0,0959 x$ & 0,99 \\
\hline & $\mathrm{S}$ acumulado $\left(\mathrm{kg} \mathrm{ha}^{-1}\right)$ & 5,3 & 8,9 & 12,1 & 18,7 & $* * *$ & 26,93 & $y=5,4003+0,6694 x$ & 0,99 \\
\hline
\end{tabular}

${ }^{1}$ Massa hectolítrica de grãos; ns = não significativo; * significativo a $P<0,10 ; * *$ significativo a $P<0,05 ; * * *$ significativo a $P<0,01 ; * * * *$ significativo a $P<0,001$. 
A produção de grãos, o teor total de $\mathrm{S}$ e o acúmulo de $\mathrm{S}$ nos grãos de trigo não foram afetados pela aplicação das doses desse nutriente (Tabela 2). A produtividade média foi de 2,36 $\mathrm{tha}^{-1}$, com teor médio de $\mathrm{S}$ nos grãos de $0,99 \mathrm{~g} \mathrm{~kg}^{-1}$, exportando-se somente $2,36 \mathrm{~kg}^{\mathrm{de}} \mathrm{S} \mathrm{ha}^{-1}$. A produção de trigo obtida situou-se acima da faixa média para a região de Santa Maria (1,77 $\left.\mathrm{t} \mathrm{ha}^{-1}\right)$ e acima da faixa média colhida no Rio Grande do Sul $\left(1,81 \mathrm{t} \mathrm{ha}^{-1}\right)$, entre os anos de 2001 e 2004 (Reunião da Comissão Sul-Brasileira de Pesquisa de Trigo, 2005). A massa hectolítrica (MH) dos grãos de trigo também não foi alterada pela adubação sulfatada, sendo que a média obtida foi de $79 \mathrm{~kg} 100$ $\mathrm{L}^{-1}$. Da mesma forma, Rheinheimer et al. (2005) e Osório Filho et al. (2007) não encontraram respostas do trigo à aplicação de doses crescentes de $\mathrm{S}$ na semeadura da cultura, sendo que uma das possíveis causas da ausência de respostas do trigo nesses trabalhos pode ter sido a rápida lixiviação do $\mathrm{S}$ no perfil do solo (Nogueira \& Melo, 2003; Osório Filho et al., 2009) para profundidades abaixo da zona de absorção radicular, antes que a cultura tivesse se estabelecido plenamente. Porém, convém relatar que mesmo fornecendo o $\mathrm{S}$ no momento de maior necessidade da cultura (no início da floração do trigo), como foi o caso deste trabalho, não foi observado incremento na produção. Dessa forma, mesmo com teores de $\mathrm{S}$ no solo abaixo do nível de suficiência para o trigo (5 mg dm${ }^{-3}$, segundo a CQFS-RS/SC, 2004), pode-se inferir que a quantidade de $\mathrm{S}$ nativo no solo e/ou depositado pela água das chuvas foi suficiente para suprir as necessidades da cultura do trigo para o seu bom crescimento e desenvolvimento.

A produção de matéria seca da parte aérea do milheto não foi afetada pela aplicação das doses de $\mathrm{S}$, apresentando produção média de 7,23 t ha-1 (Tabela 2), mesmo com teores de $\mathrm{S}$ disponível abaixo do nível crítico estabelecido pela CQFS-RS/SC (2004) (5 $\mathrm{mg} \mathrm{dm}^{-3}$ para as gramíneas). Já os teores e o acúmulo de $\mathrm{S}$ na parte aérea aumentaram linearmente com as doses de S aplicadas, sendo que no tratamento com a maior dose esses valores equivaleram, respectivamente, a 3,7 e 3,5 vezes os valores encontrados no tratamento testemunha. Considerando-se o acúmulo de $\mathrm{S}$ na parte aérea do milheto da testemunha como a quantidade de $\mathrm{S}$ nativo do solo e/ou depositado pela atmosfera que a planta pode absorver (5,3 $\left.\mathrm{kg} \mathrm{ha}^{-1}\right)$, e subtraindo-se esse valor da quantidade de $\mathrm{S}$ acumulado nos demais tratamentos, as quantidades de $\mathrm{S}$ resultantes $\left(3,6 ; 6,8\right.$ e 13,4 $\mathrm{kg}$ de $\left.\mathrm{S} \mathrm{ha}^{-1}\right)$ representam 72, 68 e $67 \%$ do $\mathrm{S}$ adicionado pelo sulfato de cálcio nos tratamentos com 5,10 e $20 \mathrm{~kg} \mathrm{ha}^{-1}$ de S, respectivamente. Isso evidencia o importante papel reciclador de $\mathrm{S}$ dessa cultura, pois o que estava acumulado na matéria seca do milheto passou a compor as formas potencialmente disponíveis no solo para os cul- tivos seguintes. Dessa forma, sistemas de produção agrícola com rotação de culturas que utilizam plantas de cobertura com crescimento radicular agressivo, como o feijão-de-porco e o milheto, maximizam a biociclagem do $\mathrm{S}$, diminuindo suas perdas no sistema solo-planta-atmosfera.

Pelos resultados das análises de regressão mostrados na Tabela 2, a maior produção de grãos da mamona nos dois cultivos, e a maior produção de matéria seca do segundo cultivo de feijão-de-porco foram obtidas com aplicação de $14,5 \pm 0,35 \mathrm{~kg} \mathrm{ha}^{-1}$ de S. Apesar disso, a resposta em produção desses cultivos foi mais relacionada com a aplicação de $\mathrm{S}$ do que com as doses aplicadas. A aplicação de apenas $5 \mathrm{~kg} \mathrm{ha}^{-1}$ de $\mathrm{S}$ foi suficiente para elevar a produção desses cultivos a valores semelhantes aos obtidos com as maiores doses de $\mathrm{S}$ aplicadas (10 e $20 \mathrm{~kg}$ ha${ }^{1}$ de $\mathrm{S}$ - Tabela 2), sendo que a aplicação de doses maiores não implicou aumento da produção, mas sim "consumo de luxo" de S (Marschner, 1995), pois as plantas absorveram quantidades do nutriente maiores que suas necessidades e as acumularam em seus tecidos.

O monitoramento da entrada de $\mathrm{S}$ atmosférico, no período de setembro de 2007 a abril de 2009, demonstrou que existe ampla variação na concentração de $\mathrm{SO}_{4}^{-}$ ${ }^{2}$ na água das chuvas, ao longo do tempo (Figura 1). A maior concentração de $\mathrm{SO}_{4}^{-2}$ ocorreu nas precipitações do mês de março de 2008 (1,67 $\left.\mathrm{mg} \mathrm{L}^{-1}\right)$ e, a menor, nas precipitações do mês de dezembro de 2008 (0,34 mg L${ }^{1}$ ), sendo que a concentração média de todo o período avaliado foi de $0,98 \mathrm{mg} \mathrm{L}^{-1}$. A chuva pode ser considerada um "sistema de limpeza" da atmosfera (Osório Filho et al., 2007). Em locais com baixa influência da contribuição de $\mathrm{S}$ atmosférico oriundo do aerosol marinho e da atividade vulcânica, como a região central do Rio Grande do Sul, a concentração de $\mathrm{SO}_{4}^{-2}$ na água da chuva é influenciada, principalmente, pela quantidade de poluentes atmosféricos, que, por sua vez, varia conforme a movimentação das massas de ar, resultando, assim, em grande oscilação nos teores de $\mathrm{SO}_{4}{ }^{-2}$ na água das chuvas, ao longo do ano. A média mensal de $\mathrm{S}$ depositado no solo pela água da chuva foi de $0,37 \mathrm{~kg}$ $\mathrm{ha}^{-1} \mathrm{mês}^{-1}$, que equivale a $4,5 \mathrm{~kg}$ de $\mathrm{S} \mathrm{ha}^{-1} \mathrm{ano}^{-1}$. De acordo com os teores médios de $\mathrm{S}$ encontrados nos grãos de trigo $\left(0,99 \mathrm{~g} \mathrm{~kg}^{-1}\right)$ e de mamona $\left(1,32 \mathrm{~g} \mathrm{~kg}^{-1}\right)$, o aporte de $\mathrm{S}$ atmosférico pela água das chuvas seria suficiente para a produção de $4.455 \mathrm{~kg}$ de grãos de trigo ou de $3.409 \mathrm{~kg}$ de grãos de mamona. Isso equivale a 1,9 vezes a produção média do trigo, ou a 2,2 ou 3,8 vezes a produção média do primeiro e segundo cultivos da mamoneira deste trabalho, respectivamente. Dessa forma, a deposição atmosférica de S é suficiente para repor ao solo a quantidade do nutriente exportada pelo trigo ou pela mamoneira por mais de uma safra. 


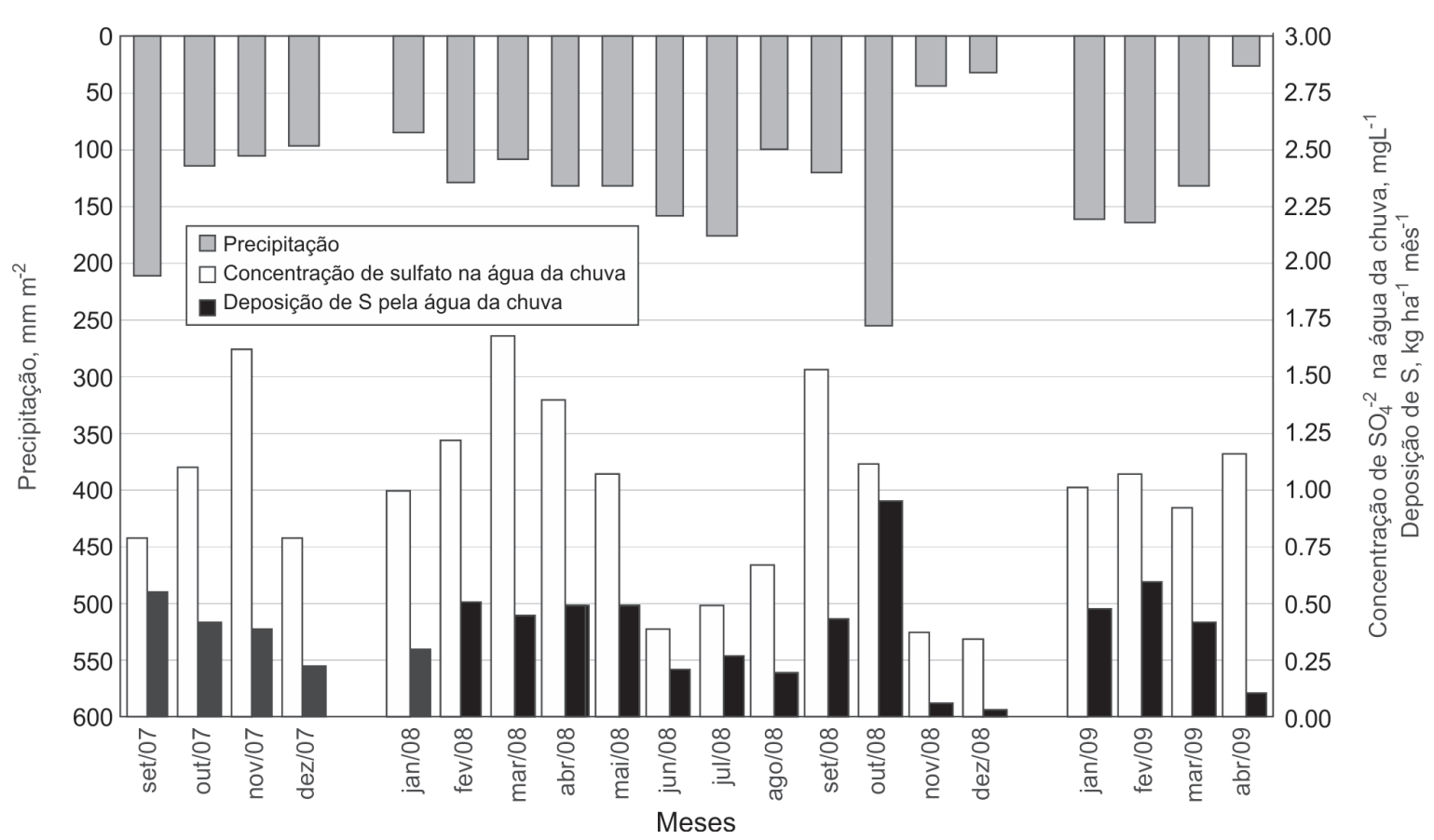

Figura 1. Precipitação, concentração de S e deposição de S pela água da chuva no período de setembro de 2007 a abril de 2009.

\section{CONCLUSÕES}

A maior produção de grãos da mamona e de matéria seca do segundo cultivo de feijão-de-porco foi obtida com aplicação de $14,5 \pm 0,35 \mathrm{~kg} \mathrm{ha}^{-1}$ de $\mathrm{S}$ no solo, mas a resposta em produção desses cultivos foi mais relacionada com a aplicação de $\mathrm{S}$ do que com as doses aplicadas.

$\mathrm{O}$ incremento nas doses de $\mathrm{S}$ adicionado ao solo proporciona aumentos lineares ou quadráticos no teor e na quantidade acumulada de $\mathrm{S}$ na parte aérea do feijão-deporco e milheto, assim como no acúmulo desse nutriente nos grãos de mamona.

Mesmo com teores de enxofre disponível no solo abaixo dos níveis de suficiência propostos pela Comissão de Química e Fertilidade do Solo dos Estados do Rio Grande do Sul e Santa Catarina, não houve respostas do trigo, do milheto e do primeiro cultivo de feijão-de-porco à adubação sulfatada.

A quantidade de enxofre atmosférico depositada no solo pela água da chuva foi de $4,5 \mathrm{~kg} \mathrm{ha}^{-1} \mathrm{ano}^{-1}$ e pode ter contribuído para a ausência de respostas de alguns cultivos à adubação sulfatada.

\section{REFERÊNCIAS}

Araujo TJ (2011) Deposição atmosférica total de nitrogênio e íons maiores no trecho inferior do rio cachoeira, sul da Bahia Brasil. Dissertação de Mestrado. Universidade Estadual de Santa Cruz, Ilhéus. 89p.

Barraco M, Díaz-Zorita M, Brambilla C, Álvarez, C \& Scianca C (2009) Respuesta del trigo a la fertilización nitrogenada y nitroazufrada en suelos arenosos. Ciencia del Suelo, 27:217-224.
Brady NC \& Weil RR (2002) The nature and properties of soils. 13ed. New Jersey, Prentice-Hall. 960p.

Comissão de Química e Fertilidade do Solo - RS/SC (2004) Manual de adubação e calagem para os estados do Rio Grande do Sul e Santa Catarina. 10. ed. Porto Alegre, Sociedade Brasileira de Ciência do Solo - Núcleo Regional Sul. 400p.

Crusciol CAC, Soratto RP, Silva LM \& Lemos LB (2006) Aplicação de enxofre em cobertura no feijoeiro em sistema de plantio direto. Bragantia, 65:459-465.

Duggan TM, Rodriguez MB, Lavado RS \& Melgar R (2010) Eficiencia agronómica del azufre elemental relativa a una fuente azufrada soluble en trigo en la Región Pampeana. Ciencia del Suelo, 28:67-77.

Empresa Brasileira de Pesquisa Agropecuária - EMBRAPA (2006) Centro Nacional de Pesquisa de Solos. Sistema brasileiro de classificação de solos. Rio de Janeiro, Embrapa Solos. 306p.

Essington ME (2005) Soil and water chemistry: an integrative approach. London, CRC Press. 534p.

Fageria VD (2001) Nutrient interactions in crop plants. Journal of Plant Nutrition, 24:1269-1290.

Guedes RMM (1985) Enxofre e nitrogênio na água da chuva e enxofre atmosférico na região metropolitana de Porto Alegre (RS), Brasil. Dissertação de Mestrado. Universidade Federal do Rio Grande do Sul, Porto Alegre. 117p.

Ishiguro M \& Makino T (2011) Sulfate adsorption on a volcanic ash soil (allophanic Andisol) under low pH conditions. Colloids and Surfaces A: Physicochemical and Engineering Aspects, 384:121-125.

Jung K, Ok YS \& Chang SX (2011) Sulfate adsorption properties of acid-sensitive soils in the Athabasca oil sands region in Alberta, Canada. Chemosphere, 84:457-463.

Lavres Junior J, Boaretto RM, Silva MLS, Correia D, Cabral CP \& Malavolta E (2005) Deficiências de macronutrientes no estado nutricional da mamoneira cultivar Iris. Pesquisa Agropecuária Brasileira, 40:145-151. 
Lavres Junior J, Cabral CP, Rossi ML, Nogueira TAR, Nogueira NL \& Malavolta E (2012) Deficiency symptoms and uptake of micronutrients by castor bean grown in nutrient solution. Revista Brasileira de Ciência do Solo, 36:233-242.

Marschner H (1995) Mineral nutrition of higher plants. New York: Academic Press. 889p.

Moterle DF, Kaminski J, Rheinheimer DS, Silva LS, Brunetto G, Tiecher T \& Fries HM (2007) Adubação potássica na cultura da mamoneira em solo arenoso sob sistema plantio direto na região central do Rio Grande do Sul. Informe Técnico 8. Universidade Federal de Santa Maria, Santa Maria 5p.

Nogueira MA \& Melo WJ (2003) Enxofre disponível para a soja e atividade de arilsulfatase em solo tratado com gesso agrícola. Revista Brasileira de Ciências do Solo, 27:655-663.

Osório Filho BD, Rheinheimer DS, Dias GF, Kaminski J, Silva LS, Brunetto G \& Casali CA (2009) Flutuação dos teores de sulfato na solução do solo em cultivos de feijoeiro e canola. Revista Brasileira de Agrociência, 15:71-75.

Osório Filho BD, Rheinheimer DS, Silva LS, Kaminski J \& Dias GF (2007) Deposição do enxofre atmosférico no solo pelas precipitações pluviais e respostas de culturas à adubação sulfatada em sistema plantio direto. Ciência Rural, 37:712-719.

Osório Filho BD, Rheinheimer DS, Dias GF, Kaminski J, Silva LS, Brunetto G \& Casali CA (2009) Flutuação dos teores de sulfato na solução do solo em cultivos de feijoeiro e de canola. Revista Brasileira Agrociência, 15:71-75.

Pozza AAA, Curi N, Guilherme LRG, Marques JJGSM, Costa ETS, Zuliani DQ, Motta PEF, Martins RS \& Oliveira LCA (2009) Adsorção e dessorção aniônicas individuais por gibbsita pedogenética. Química Nova, 32:99-105.

Reunião da Comissão Sul-Brasileira de Pesquisa de Trigo (2005) Indicações técnicas da Comissão Sul brasileira de Pesquisa de Trigo. Passo Fundo: Embrapa Trigo. 162p.
Rheinheimer DS, Alvarez JWR, Osório Filho BD, Silva LS \& Bortoluzzi, EC (2005) Resposta de culturas à aplicação de enxofre e a teores de sulfato num solo de textura arenosa sob plantio direto. Ciência Rural, 35:562-569.

Salvagiotti F, Castellarín JM, Miralles DJ \& Pedrol HM (2009) Sulfur fertilization improves nitrogen use efficiency in wheat by increasing nitrogen uptake. Field Crops Research, 113:170177.

Severino LS, Ferreira GB, Moraes CRA, Gondim TMS, Freire WSA, Castro DA, Cardoso GD \& Beltrão NEM (2005) Adubação química da mamoneira com macro e micronutrientes em Quixeramobim, CE. Campina Grande, PB. Embrapa Algodão. Boletim de Pesquisa e Desenvolvimento, 61. 23p.

Severino LS, Ferreira GB, Moraes CRA, Gondim TMS, Cardoso GD, Viriato JR \& Beltrão NEM (2006) Produtividade e crescimento da mamoneira em resposta à adubação orgânica e mineral. Pesquisa Agropecuária Brasileira, 41:879-882.

Silva MA, Silva FEA, Nunes Júnior ES, Costa FX \& Melo Filho JS (2012) Cultivo de sequeiro da mamona adubada com casca de mamona e fertilizante nitrogenado. Revista Brasileira de Engenharia Agrícola e Ambiental, 16:375-379.

Tabatabai MA \& Bremner JM (1970) An alkaline oxidation method for determination of total sulphur in soils. Soil Science Society of America Proceedings, 34:62-65.

Tedesco MJ, Gianello C, Bissani CA, Bohnen H \& Volkweiss SJ (1995) Análise de solo, plantas e outros materiais. Boletim Técnico de Solos 5. Universidade Federal do Rio Grande do Sul, Porto Alegre. 215p. 